\title{
Quantum correlations in position, momentum, and intermediate bases for a full optical field of view
}

\author{
J. Leach, ${ }^{1}$ R. E. Warburton, ${ }^{2}$ D. G. Ireland, ${ }^{1}$ F. Izdebski, ${ }^{2}$ S. M. Barnett, ${ }^{3}$ A. M. Yao, ${ }^{3}$ G. S. Buller, ${ }^{2}$ and M. J. Padgett ${ }^{1}$ \\ ${ }^{1}$ SUPA, School of Physics and Astronomy, University of Glasgow, Glasgow G12 8QQ, United Kingdom \\ ${ }^{2}$ SUPA, School of Engineering and Physical Sciences, Heriot-Watt University, Edinburgh EH14 4AS, United Kingdom \\ ${ }^{3}$ SUPA, Department of Physics, University of Strathclyde, Glasgow G4 ONG, United Kingdom
}

(Received 26 July 2011; published 20 January 2012)

\begin{abstract}
We report an eight-element, linear-array, single-photon detector that uses multiple fibers of differing lengths coupled to a single detector, the timing information from which reveals the position in which the photon was measured. Using two such arrays and two detectors we measure the correlations of photons produced by parametric downconversion, without recourse to mechanical scanning. Spatial light modulators acting as variable focal length lenses positioned between the downconversion crystal and the arrays allow us to switch between measurement of position, transverse momentum, or intermediate bases. We observe the product of the variances of the conditional probabilities for position and momentum to be more than an order of magnitude below the classical limit, realizing a full-field demonstration of the Einstein-Podolsky-Rosen paradox. Such, multistate measurement technologies allow access to the higher information content of the photon based upon spatial modes.
\end{abstract}

DOI: 10.1103/PhysRevA.85.013827

PACS number(s): 42.50.Ar, 03.65.Ud, 29.40.Gx

\section{INTRODUCTION}

Pairs of photons produced by parametric downconversion are commonly used to investigate and apply quantum entanglement. Although the concept of spatial entanglement was introduced in the original Einstein-Podolsky-Rosen paradox (EPR) paper of 1935 [1], it was not until the work of Howell et al. in 2004 that correlations were strong enough to reach the EPR limit [2]. In that work, strong correlations were observed, both in the image plane of the downconversion crystal (associated with the position basis) and in the far-field of the crystal (associated with the momentum basis). In parallel with that work, studies were also completed using an additional beam splitter so that the image and far-field planes could be scanned without changing the optical configuration [3]. In these previous works the coincidence count, as a function of lateral position, was obtained sequentially by scanning a single detector across the field of view [2] or sequentially connecting a detector into fibers located at different positions [4]. Interpreting that experiment as a realization of EPR required the reasonable assumption that the light behaves in the same way irrespective of which fiber is being monitored. However, beyond this assumption and potential implications for EPR, scanning techniques have a more serious limitation. If the spatial modes of the photon are to be used to increase its information capacity, a measurement technique is required which has a multistate output. By contrast a single, scanning measurement is a one bit system; i.e., in any measurement window, the photon is detected or not.

The ideal detection scheme would measure the full optical field of view in the image or far-field plane, detecting all modes with equal quantum efficiency. Increasing the number of individual detectors requires an increasingly sophisticated measurement system and one is left with the option of designing a complicated multi-way coincidence detection scheme [5], using arrays of single-photon detectors [6] or electron-multiplying or intensified cameras [7,8]. To date, no multielement detector has shown single-photon quantum correlations of a strength sufficient to demonstrate positionmomentum EPR.

\section{EXPERIMENT}

In this paper, we report full-field quantum correlations in the image, far-field, and intermediate planes of the entangled photon pairs emitted from a downconversion crystal. We make these observations using two, eight-fiber arrays, so realizing three bits per photon (see Fig. 1). A 30-mW, 355-nm-wavelength pump laser with an output beam diameter of $1 \mathrm{~mm}$ is incident upon a 3 -mm-long type- $1, \beta$-barium borate (BBO) crystal that produces colinear downconverted photon pairs at a wavelength of $710 \mathrm{~nm}$. The exit face of the crystal is reimaged, using an afocal telescope with a magnification of 5, through a 50:50 beam splitter giving two optical arms and two separated images of the crystal. In each arm, the fiber array is placed a distance of $800 \mathrm{~mm}$ from this image of the crystal, marked as distance of $2 d$ in Fig. 1. Positioned midway between the reimaged crystal and the fiber arrays is the combination of a 400-mm focal length lens and a spatial light modulator (SLM, Hamamatsu LCOS). The SLM can be programed to provide a predetermined level of optical power in the first diffracted order. When the SLM provides no additional optical power, the $400-\mathrm{mm}$ focal length lens alone means that the fiber arrays are in the far-field of the crystal. Alternatively, when the SLMs are programed as $f \sim$ $400 \mathrm{~mm}$ lenses, then in conjunction with the 400-mm lens the fiber arrays are in the image plane of the crystal. Both fiber arrays comprise eight fibers with a 50- $\mu \mathrm{m}$ core diameter, spaced with a $127-\mu \mathrm{m}$ pitch (Oz Optics). The eight fibers from one array are combined with a $105-\mu \mathrm{m}$ core fiber and connected to a single-photon avalanche diode (SPAD) and the eight fibers from the other array are connected to a second SPAD. The optical efficiency of the fiber combiners exceed $70 \%$ [9], meaning that any additional losses in our technique are more than compensated for by the eightfold increase in the number of measurement states. A detection from one 


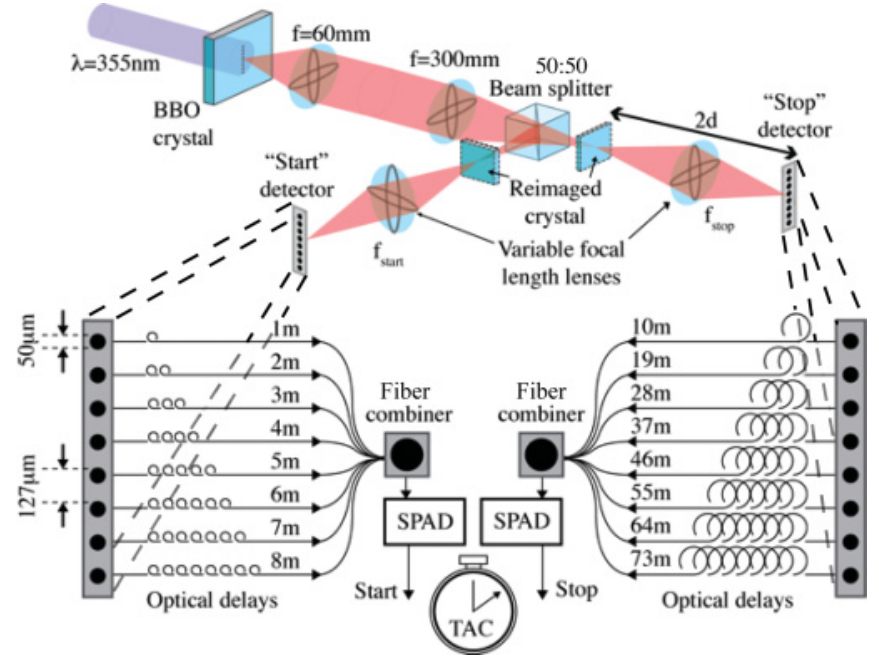

FIG. 1. (Color online) Schematic arrangement of the downconversion source relayed to either the image plane or far-field onto two eight-element fiber arrays. Each array is coupled to a single-photon detector via differing lengths of optical delay. The overall delay between the start and stop triggers allows identification of the position of both detected photons.

array starts the time-to-amplitude converter (TAC), and the second stops it. The 64 possible combinations of delay times identify uniquely the entrance fiber of both photons. (A similar rational has been used successfully for converting wavelength information into a temporal signature [10]). For the eight fiber arrays the length increments of the optical fibers correspond to timing delays of $\Delta t_{i}=4.9 \mathrm{~ns}$ and $\Delta t_{j}=44.1 \mathrm{~ns}$, where the subscripts $i$ and $j$ refer to start and stop, respectively. A slight timing jitter of the SPAD detectors means that we set the width of the bin at $\delta t=2.5 \mathrm{~ns}$.

\section{RESULTS AND DISCUSSION}

In terms of demonstrating a quantum effect we face a number of issues. First, as with all experiments of this type, some coincidence counts can arise from accidental correlations that are not of a quantum origin. Second, the fixed spacing of a finite number of optical fibers means that the measurements are sampled both at discrete positions and only over a finite range. Any approach to calculating the standard deviation of the width of the observed correlations requires some method to deal with these technical limitations.

If the count rates from the two SPAD detectors are $S_{i}$ and $S_{j}$ this gives a baseline level of coincidences, $B=S_{i} S_{j} \delta t$, which occurs independently of any quantum correlations. For our experiment these count rates are 6000 and 9000 counts per SPAD in momentum and position measurements, respectively, giving accidental coincidence rates of $B_{p}=0.1 / \mathrm{s}$ and $B_{x}=$ 0.2 /s per time bin for momentum and position, respectively.

Figures 2(a) and 2(b) show the coincidence counts in both the position (image) and momentum (far-field) planes as measured by the eight fibers in the two arms summed over 30 minutes. When placed in an image plane of the crystal, the sampling interval of the position measurement is simply that of the fiber spacing, i.e., $\delta x=0.127 \mathrm{~mm}$. When placed in the far-field plane, the corresponding interval in (a) Image correlations

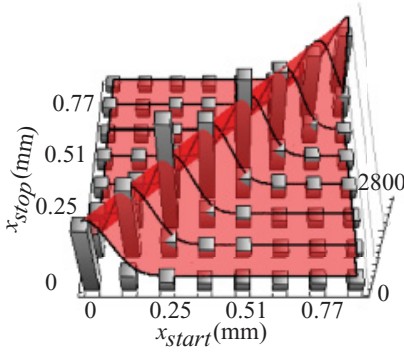

(b) Far-field correlations

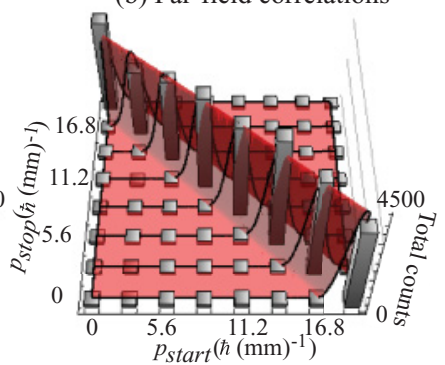

FIG. 2. (Color online) The full-field correlations in both the image (left) and far-field (right) planes. A baseline offset Gaussian surface is fitted to these observations giving an estimate of the standard deviation of both position and momentum correlations from which an indicative demonstration of EPR can be made.

sampling of the transverse momentum is $\delta p_{x}=(\delta x / f) \hbar k_{0}$, where $f=400 \mathrm{~mm}$ is the focal length of the coupling lens and $k_{0}$ is the wave number of the downconverted light, giving $\delta p_{x}=2.8 \hbar(\mathrm{mm})^{-1}$. The resulting surface plot for the coincidence counts, $C$, is proportional to the modulus squared of the overlap integral between the measured states and the two-photon wave function describing the downconverted light [11]. The ratio of the correlation peaks to those of the accidentals is approximately 20:1.

Given that the profile of the pump beam is Gaussian, we would anticipate that the form of the observed correlation will be approximately Gaussian and also that the overall count rates will have a Gaussian profile across the fiber array [11,12]. Adopting the analysis of Ref. [12], based on a calculation of the two-photon wave function, we can write the anticipated coincidence count rates in the position and momentum planes as, respectively,

$$
\begin{aligned}
C_{x}\left(x_{i}, x_{j}\right)= & A_{x}\left[\exp \left(\frac{-\left(x_{i}-x_{j}\right)^{2}}{2 \sigma_{x}^{2}}\right)\right. \\
& \left.\times \exp \left(\frac{-\sigma_{p}^{2}\left(x_{i}+x_{j}\right)^{2}}{2 \hbar^{2}}\right)\right]+B_{x}
\end{aligned}
$$

and

$$
\begin{aligned}
C_{p}\left(p_{i}, p_{j}\right)= & A_{p}\left[\exp \left(\frac{-\left(p_{i}+p_{j}\right)^{2}}{2 \sigma_{p}^{2}}\right)\right. \\
& \left.\times \exp \left(\frac{-\sigma_{x}^{2}\left(p_{i}-p_{j}\right)^{2}}{2 \hbar^{2}}\right)\right]+B_{p} .
\end{aligned}
$$

The width of the position and momentum correlations are anticipated [11] to be functions of the magnified crystal length, $L$, the size of the pump beam, $w_{\text {pump}}$, and the magnification between the crystal and the fiber array, $M$. The standard deviations of these correlations are given by $\sigma_{x}=M \sqrt{L \lambda_{\text {pump }} / 2 \pi}$ and $\sigma_{p}=\hbar / M w_{\text {pump }}$. This would predict underlying correlations of $\sigma_{x}=0.065 \mathrm{~mm}$ and $\sigma_{p}=$ $0.4 \hbar(\mathrm{mm})^{-1}$. We emphasize, however, that our demonstration of EPR does not rely on this specific model.

Fitting our data to these equations gives $\sigma_{x}=0.101 \pm$ $0.007 \mathrm{~mm}$ and $\sigma_{p}=(1.24 \pm 0.11) \hbar(\mathrm{mm})^{-1}$. In both cases these measured values are somewhat broader than those predicted by theory. We attribute this additional width to 
a slight undersampling of the downconverted light by the discrete nature of the fiber arrays.

In order to demonstrate the EPR paradox, it is sufficient to show that the product of the two variances, $\Delta^{2}\left(x_{i}-x_{j}\right)$ and $\Delta^{2}\left(p_{i}+p_{j}\right)$, is less than $\hbar^{2} / 4$ [13-15]. From the fitted data we find the position-difference variance to be

$$
\Delta^{2}\left(x_{i}-x_{j}\right)=1.02 \times 10^{-2} \pm 0.10 \times 10^{-2} \mathrm{~mm}^{2},
$$

and we find the corresponding variance for the momentum sum to be

$$
\Delta^{2}\left(p_{i}+p_{j}\right)=(1.53 \pm 0.20) \hbar^{2}(\mathrm{~mm})^{-2} .
$$

The variance product we obtain is $(0.016 \pm 0.003) \hbar^{2}$, over 1 order of magnitude below the classical limit.

Although positioning the fiber arrays in the image or farfield planes corresponds directly to measurement of position or momentum, respectively, correlations between the photon pairs are not restricted to these planes alone [12]. For any system configuration the size of the coincidence count between the two detectors can be calculated from the two-photon wave function. It is more appealing physically, however, to take advantage of the Klyshko interpretation, which allows us to predict the observed quantum correlation using only classical optics [16]. In this analogous classical system the nonlinear crystal is replaced with a mirror and one of the two detectors acts as a source. The size of the coincidence count can be predicted from the transmission coefficient between source and detector, now separated from each other by $4 d$. We should emphasize, however, that this classical analogy is simply a convenient predictive tool rather than a physical model. It can be related, nevertheless, to the retrodictive formulation of quantum theory $[17,18]$. Both the formal quantum theory and the Klyshko picture predict that the correlation signal should be high whenever the detector planes are imaged onto each other. The condition for this detector-to-detector imaging is

$$
f_{i}=\left(\frac{1}{d}+\frac{1}{2 d-\left(1 / f_{j}-1 / d\right)^{-1}}\right)^{-1},
$$

giving a magnification of

$$
M\left(f_{i}\right)=\frac{\left(1 / f_{i}-1 / d\right)^{-1}}{2 d-\left(1 / f_{i}-1 / d\right)^{-1}} .
$$

The image $\left(f_{i}=f_{j}=d / 2\right)$ and far-field planes $\left(f_{i}=f_{j}=d\right)$ are just special, symmetrical examples of this condition where the magnification between detector planes is +1 and -1 , respectively. Symmetry between the two arms is not essential, and strong correlations are predicted for other magnifications too. These spatial correlations have been used to investigate quantum correlations for spatial states. [12,19]. Figure 3 shows the total coincidence rate (summed over all 8 fibers) as a function of the focal lengths of the two SLMs. We see that the coincidence rates are high whenever this back projection imaging condition is satisfied.

Figure 4 shows the individual coincidence rates (for each combination of fibers) for the special cases of where the magnification of the back projection imaging corresponds to an integer or rational fraction. Beyond the strong correlations corresponding to magnifications of +1 (image) and -1 (far-field) one notes that strong correlations arise from

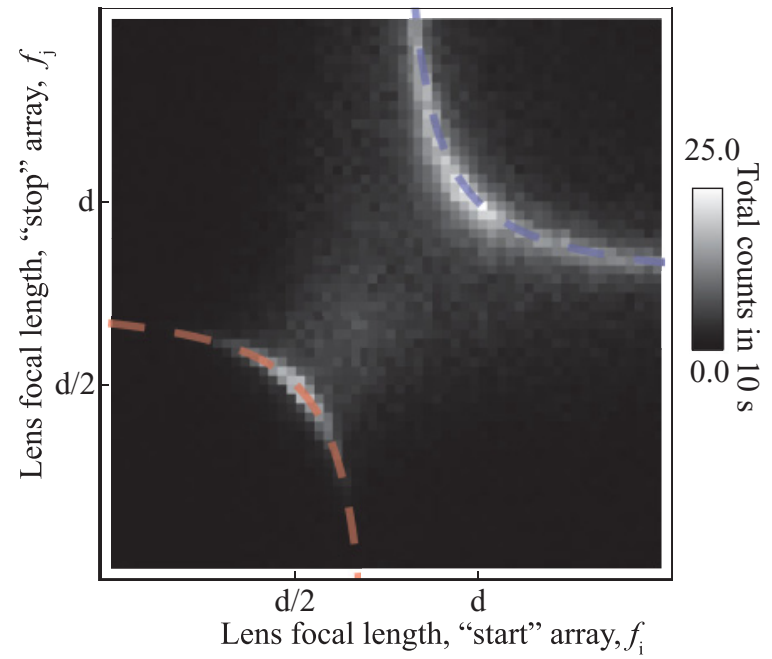

FIG. 3. (Color online) The coincidence count rate summed over all eight fibers as a function of the focal length in start and stop optical paths.
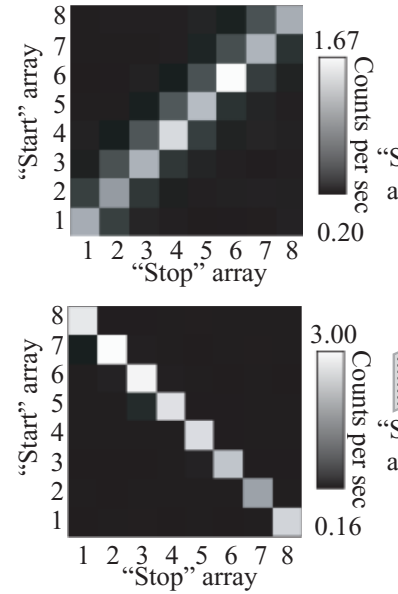

(a) Image plane correlations, $\mathrm{M}=1$

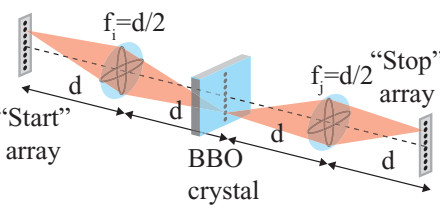

(b) Far-field correlations, $\mathrm{M}=-1$

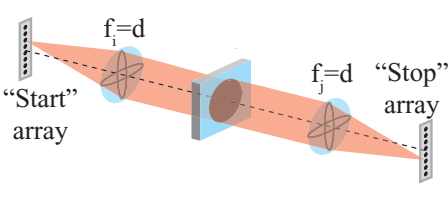

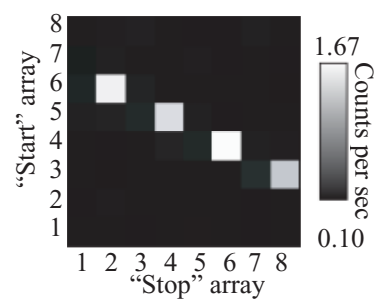

(c) Intermediate-field correlations, $\mathrm{M}=-2$
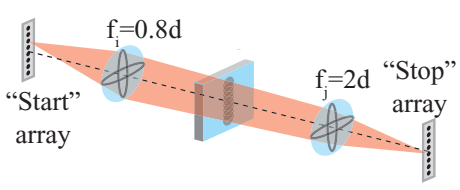

(d) Intermediate-field correlations, $M=-1 / 2$

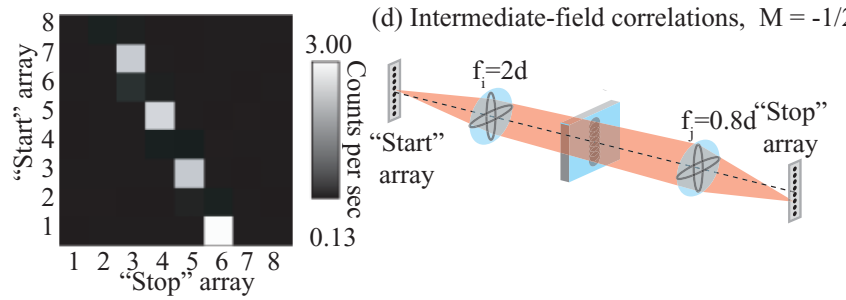

FIG. 4. (Color online) The details of the (anti)correlations observed for various focal lengths. A magnification of +1 corresponds to both detector arrays being in the image plane of the crystal, and a magnification of -1 corresponds to when both detectors are in the far-field of the crystal. Magnifications of -2 and $-1 / 2$ are shown also. 
other magnifications too. For example, for the cases of a magnification between the detector arrays of -2 or $-1 / 2$, each fiber in one array shows a strong correlation with every alternate fiber in the other.

\section{CONCLUSION}

The novelty of this work is the demonstration of an eightstate simultaneous measurement for each of the two photons in an entangled pair. The key benefit, however, is that our technique allows us to access an eight-dimensional state space for each of the downconverted photons. The use of SLMs acting as variable focal length lenses allows the measurements to be switched between position, momentum, and intermediate bases. A potential application of this would be the realization of quantum-information processing or communication [20-23]. This use of spatial states could be combined with other degrees of freedom to extend yet further the information capacity of the photon.

\section{ACKNOWLEDGMENTS}

M.J.P. and S.M.B. thank the Royal Society and the Wolfson Foundation. We acknowledge the financial support of the UK EPSRC and the Future and Emerging Technologies (FET) program within the Seventh Framework Programme of the European Commission, under agreement HIDEAS No. FP7-ICT-221906. We thank Hamamatsu for support of this work and Daniel Tasca for helpful discussions.
[1] A. Einstein, B. Podolsky, and N. Rosen, Phys. Rev. 47, 777 (1935).

[2] J. C. Howell, R. S. Bennink, S. J. Bentley, and R. W. Boyd, Phys. Rev. Lett. 92, 210403 (2004).

[3] M. D'Angelo, Y.-H. Kim, S. P. Kulik, and Y. Shih, Phys. Rev. Lett. 92, 233601 (2004).

[4] M. N. O’Sullivan-Hale, I. A. Khan, R. W. Boyd, and J. C. Howell, Phys. Rev. Lett. 94, 220501 (2005).

[5] A. Peruzzo et al., Science 329, 1500 (2010).

[6] G. S. Buller and R. J. Collins, Meas. Sci. Technol. 21, 012002 (2010).

[7] L. Zhang, L. Neves, J. S. Lundeen, and I. A. Walmsley, J. Phys. B 42, 114011 (2009).

[8] A. F. Abouraddy, M. B. Nasr, B. E. A. Saleh, A. V. Sergienko, and M. C. Teich, Phys. Rev. A 63, 063803 (2001).

[9] R. E. Warburton, F. Izdebski, C. Reimer, J. Leach, D. G. Ireland, M. Padgett, and G. S. Buller, Opt. Express 19, 2670 (2011).

[10] M. Avenhaus, A. Eckstein, P. J. Mosley, and C. Silberhorn, Opt. Lett. 34, 2873 (2009).

[11] K. W. Chan, J. P. Torres, and J. H. Eberly, Phys. Rev. A 75, 050101 (2007).

[12] D. S. Tasca, S. P. Walborn, P. H. Souto Ribeiro, F. Toscano, and P. Pellat-Finet, Phys. Rev. A 79, 033801 (2009).
[13] M. D. Reid, P. D. Drummond, W. P. Bowen, E. G. Cavalcanti, P. K. Lam, H. A. Bachor, U. L. Andersen, and G. Leuchs, Rev. Mod. Phys. 81, 1727 (2009).

[14] Z. Y. Ou, S. F. Pereira, H. J. Kimble, and K. C. Peng, Phys. Rev. Lett. 68, 3663 (1992).

[15] M. D. Reid, Phys. Rev. A 40, 913 (1989).

[16] T. B. Pittman, D. V. Strekalov, D. N. Klyshko, M. H. Rubin, A. V. Sergienko, and Y. H. Shih, Phys. Rev. A 53, 2804 (1996).

[17] E.-K Tan, J. Jeffers, S. M. Barnett, and D. T. Pegg, Eur. Phys. J. D 22, 495 (2003).

[18] E.-K. Tan, J. Jeffers, S. M. Barnett, and D. T. Pegg, Eur. Phys. J. D 29, 309 (2004).

[19] D. S. Tasca, S. P. Walborn, F. Toscano, and P. H. Souto Ribeiro, Phys. Rev. A 80, 030101 (2009).

[20] M. P. Almeida, S. P. Walborn, and P. H. Souto Ribeiro, Phys. Rev. A 72, 022313 (2005).

[21] I. Ali-Khan, C. J. Broadbent, and J. C. Howell, Phys. Rev. Lett. 98, 060503 (2007).

[22] S. P. Walborn, D. S. Lemelle, D. S. Tasca, and P. H. Souto Ribeiro, Phys. Rev. A 77, 062323 (2008).

[23] G. Gibson, J. Courtial, M. J. Padgett, M. Vasnetsov, V. Pas'ko, S. M. Barnett, and S. Franke-Arnold, Opt. Express 12, 5448 (2004). 